\title{
Dosage rapide par chromatographie phase gazeuse de la kétamine et de la norkétamine chez le rat
}

\section{Rapid gas chromatography determination of ketamine and norketamine in rats}

\section{Lauriane GINEFRI ${ }^{(3)}$, Bernard GOURMEL ${ }^{(3)}$, Frédéric J. BAUD ${ }^{(1,2)}$, Pascal HOUZÉ ${ }^{(1,2,3) *}$}

(1) Université Paris Descartes, Faculté de Pharmacie, Neuropsychopharmacologie des addictions, CNRS, UMR 7157 et Université Paris 7 - F-75010 Paris - France

(2) AP-HP, Hôpital Lariboisière, Réanimation Médicale et Toxicologique, 2, rue Ambroise Paré - F-75010 Paris - France (3) AP-HP, Hôpital Saint Louis, Laboratoire de Biochimie, 1, avenue Claude Vellefaux - F-75010 Paris - France

*Auteur à qui adresser la correspondance : Pascal HOUZÉ, AP-HP, Hôpital Saint Louis, Laboratoire de Biochimie, 1, avenue Claude Vellefaux - F-75010 Paris - France Tél. : +33142499446 - Fax : +33142499247 - E-mail : pascal.houze@ sls.aphp.fr

(Reçu le 2 avril 2007 ; accepté après modification le 21 septembre 2007)

\section{RÉSUMÉ}

La kétamine est un anesthésique dissociatif dérivé de la cyclohexanone, utilisée en médecine humaine et vétérinaire pour induire l'endormissement et le maintenir lors de brèves interventions chirurgicales. Inhibiteur non compétitif du glutamate au niveau des récepteurs NMDA centraux, la kétamine est rapidement métabolisée en norkétamine, présentant une action pharmacologique, puis en déhydronorkétamine éliminée par voie urinaire après conjugaison. Les effets secondaires (délires, hallucinations) sont recherchés au cours des raves parties. L'accroissement de son utilisation dans un but d'addiction est à l'origine d'un nombre élevé d'intoxications dont certaines mortelles. De nombreuses méthodes chromatographiques en phase gazeuse ont été rapportées utilisant toutes une étape de dérivation et un volume d'échantillon important. Dans ce travail, nous décrivons une méthode de dosage simultané de la kétamine et de la norkétamine par chromatographie phase gazeuse sans dérivation après extraction liquide-liquide. L'ifosfamide est utilisé

\section{SUMMARY}

Ketamine is an anaesthesic agent extensively used for the induction and maintenance of anesthesia and analgesia in man and animals. Ketamine is a NMDA receptor agonist which is metabolised in the liver in norketamine then dehydronorketamine excreted in urines as glucuroconjucated compounds. In rave party, adverse effects of ketamine are responsible of death occurring to respiratory arrest. Many gas chromatographic methods are described in literature using large sample and derivative procedure. In this paper, we report a rapid gas method without derivative step for the simultaneous determination of plasma norketamine and ketamine using ifosfamide as internal standard. The method is linear from 500 to $4.000 \mu \mathrm{g} / \mathrm{L}$ with a good sensitivity corresponding to $160 \mu \mathrm{g} / \mathrm{l}$ and $780 \mu \mathrm{g} / \mathrm{L}$ as limits detection for ketamine and norketamine, respectively. Precision (below $10 \%$ ) and accuracy (below $15 \%$ ) are satisfying with a small plasma sample. This method is available to realise pharmacologic study in rats as reported in this paper: 
comme éralon interne. La méthode est linéaire entre 500 et $4000 \mu \mathrm{g} / \mathrm{L}$. La limite de détection est de 160 et $180 \mu \mathrm{g} / \mathrm{L}$ pour la kétamine et la norkétamine, respectivement. La précision intra et inter séries est satisfaisante $(<10 \%)$, de même que l'exactitude (biais < $15 \%$ ). Utilisant un volume réduit de prélèvement, cette méthode peut être appliquée à des études pharmacocinétiques chez l'animal. Pour illustrer cette application, nous décrivons les cinétiques plasmatiques de la kétamine et de la norkétamine chez le rat après perfusion de kétamine à la dose de $70 \mathrm{mg} / \mathrm{kg}$. Les valeurs des principaux paramètres pharmacocinétiques sont également présentées.

\section{MOTS-CLÉS}

kétamine, chromatographie phase gazeuse, rat, pharmacocinétique.

\section{Introduction}

La kétamine $(\mathrm{K})$, [2-(o-chlorophenyl)-2-methylamino)] cyclohexanone (Figure 1), a été synthétisée pour la première fois en 1962, dans le but de trouver un anesthésique de remplacement à la phencyclidine (1). Utilisée sous forme de racémique, la kétamine est un agent anesthésique dissociatif à courte durée d'action (2). L'anesthésie induite se caractérise, par une analgésie profonde, le maintien des réflexes pharyngo-laryngés, la conservation du tonus musculaire et même une stimulation des fonctions cardiaque et respiratoire aux doses pharmacologiques (2). Chez l'homme, ces caractéristiques font de la kétamine (Kétalar ${ }^{\circledR}$, Kétamine Panpharma $\left.{ }^{\circledR}\right)$, un agent de choix pour induire l'anesthésie, pour les actes chirurgicaux de courte durée ou encore en analgésie dans les soins post-opératoires douloureux (3). En médecine vétérinaire, la kétamine (Kétamine UVA®, Clorketam ${ }^{\circledR}$, Imalgène ${ }^{\circledR}$ ) seule ou associée à d'autres produits (xylazine) est fréquemment utilisée pour induire et entretenir l'anesthésie dans de nombreuses espèces animales : rat, cheval, bovins (4).

D’un point de vue métabolique, la kétamine est bien absorbée par toutes les voies parentérales (90-95\%) mais faiblement per os (10-20\%) (3). La distribution tissulaire est rapide, avec une demi-vie comprise entre 7 et 11 minutes. Les concentrations les plus élevées sont retrouvées au niveau des tissus adipeux, du foie, des poumons et du cerveau, alors que les plus faibles sont observées dans les muscles et le sang circulant (3). La liaison de la kétamine aux protéines plasmatiques est controversée. Pour certains auteurs, elle serait faible, de l'ordre de $10 \%$ (5), pour d'autres elle pourrait atteindre $60 \%$ (6). Métabolisée au niveau hépatique par la voie des cytochromes P450 (principalement le CYP2B6), la kétamine conduit rapidement (demi-vie d'élimination 1 à 2 heures) à la norkétamine (NK) par déméthylation puis à des dérivés hydroxylés

\section{KEY-WORDS}

ketamine, gas chromatography, rat, pharmacokinetics.

(6- et 4- norkétamine) qui par déshydratation vont aboutir à la déhydronorkétamine (DHNK), métabolite terminal. Les dérivés hydroxylés et la DHNK sont éliminés dans les urines ( 85 à $95 \%$ de la dose administrée) après sulfo- ou glycuroconjugaison (7) (fig 1).

Sur le plan pharmacologique, la kétamine est un antagoniste non compétitif du glutamate au niveau des récepteurs $N$-methyl-D-aspartate (NMDA) centraux (8). La kétamine $S(+)$ est quatre fois plus puissante que la kétamine R (-) (9). Parmi les métabolites de la K, la norkétamine a été décrite comme pouvant interagir avec les récepteurs NDMA mais elle serait cinq fois moins active que la molécule mère. Elle pourrait cependant contribuer à l'activité pharmacologique de la kétamine, en particulier quand celle-ci est administrée par voie orale (10).

L'interaction de la $\mathrm{K}$ avec les récepteurs est bien sûr responsable de ces effets pharmacologiques mais aussi de ces effets secondaires neuropsychiques comme les hallucinations, le délire et l'agitation $(8,11)$.

Ces effets secondaires sont recherchés par les toxicomanes avérés ou occasionnels en particuliers dans les soirées rave où la kétamine circule sous différents noms (Ket, Special K, Kit Kat, Super acide,...) seule ou associée à d'autres drogues. Elle est essentiellement sniffée mais peut être consommée par voie orale ou injectée en intraveineuse. En plus de ces propriétés neuropsychiques, la kétamine a des effets désinhibiteurs et anxiolytiques très recherchés par les utilisateurs. Les effets présentent un maximum pendant 4 à 6 heures puis diminuent brusquement, entraînant un recours à d'autres drogues de remplacement, comme l'alcool ou les benzodiazépines. L'usage chronique et prolongé de la kétamine génère une tolérance et une dépendance impliquant son classement dans les produits stupéfiants en France (arrêté du 8 août 1997), mais aussi dans d'autres pays (DEA Schedule III, USA, 1999 ; Classe C, UK, 2006) (12). Le surdosage 


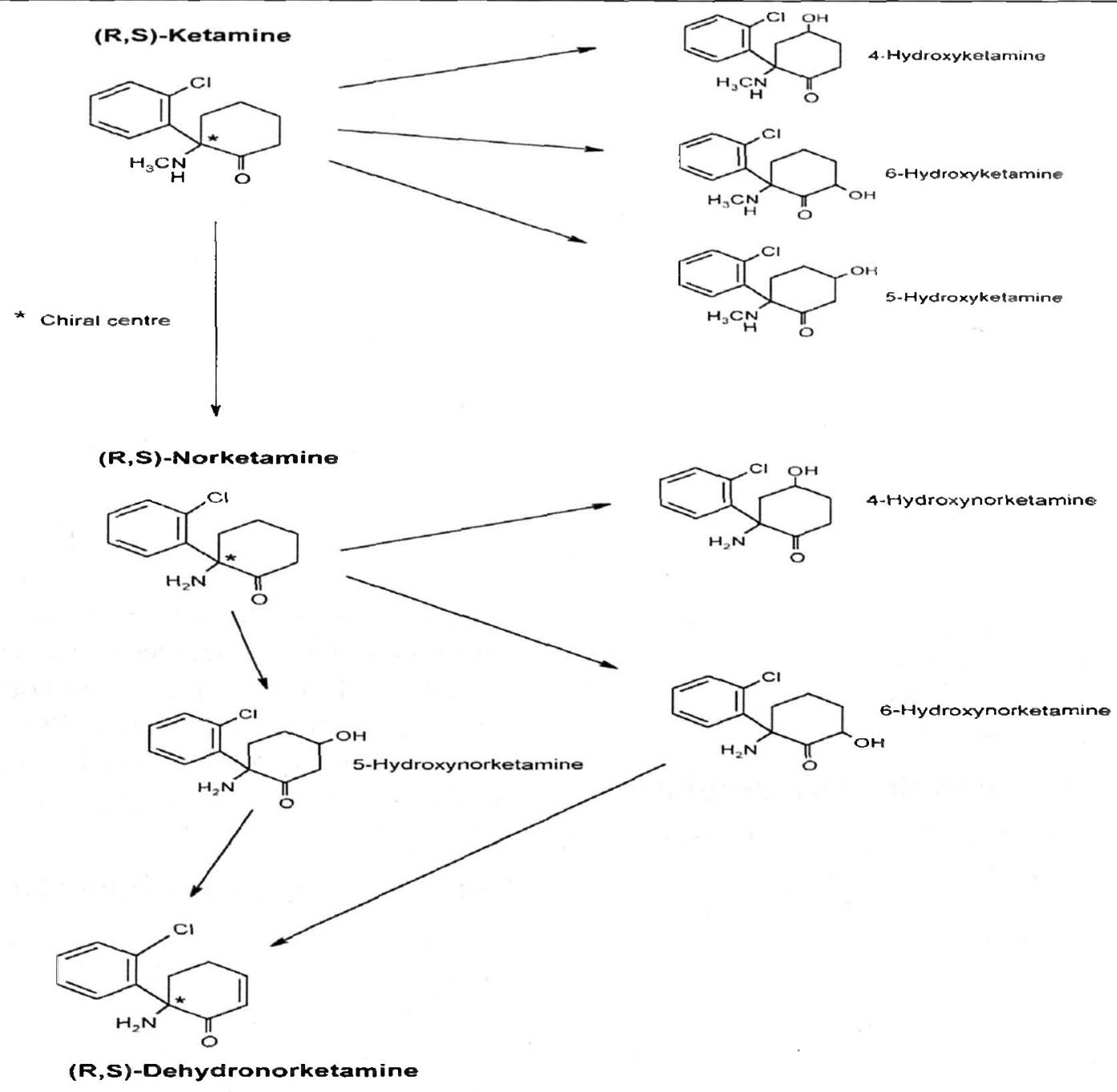

Figure 1 : Structure et métabolisme de la kétamine.

peut entraîner des convulsions, une défaillance cardiorespiratoire avec risque d'arrêt cardiaque ou une mort subite. Plusieurs cas d'intoxication fatale chez l'homme par la kétamine ont été rapportés dans la littérature $(13,14)$

Ces données toxicologiques expliquent le nouvel intérêt apporté au dosage de la kétamine et de son métabolite actif principal. D'un point de vue analytique, la revue de la littérature retrouve de nombreuses méthodes de dosage par chromatographie en phase gazeuse avec dérivation (15-17) ou en phase liquide $(4,18-19)$, nécessitant des volumes de prélèvement important (de l'ordre de $500 \mu 1$ ), difficilement applicable au petit animal lors de protocoles avec prélèvements multiples.

Dans ce travail, nous rapportons une méthode par chromatographie en phase gazeuse sans dérivation, pour le dosage simultané de la kétamine et de la norkétamine. La technique décrite est rapide, spécifique et suffisamment sensible pour travailler sur de faibles volumes d'échantillons. Compte tenu de sa sensibilité, notre méthode parait parfaitement adaptée à la réalisation d'études pharmacocinétiques chez le petit animal.

\section{Matériel et méthodes}

\section{Solvants et réactifs}

L' hydrochloride de kétamine a été obtenu auprès de Sigma-Aldrich (St Quentin Fallavier, France). L'hydrochloride de norkétamine en solution méthanolique à $1 \mathrm{~g} / \mathrm{L}$, provient de la société LGC Promochem (Molsheim, France). L'ifosfamide est un don des laboratoires Baxter (Paris, France).

Le méthanol, la lessive de soude, le chloroforme et l'acétate d'éthyle, de qualité analytique, ont été fournis par la société VWR International (Fontenay-sous-Bois, France). L'eau pour préparation injectable et le chlorure de sodium isotonique sont fournis par le Laboratoire Lavoisier (Paris, France). 
La solution mère de kétamine est préparée par dissolution du produit pesé dans du méthanol, de façon à obtenir une solution à $1 \mathrm{~g} / \mathrm{L}$ de kétamine base $(11,5 \mathrm{mg}$ d'hydrochloride de kétamine dans $10 \mathrm{~mL}$ de méthanol). La solution mère en NK est titrée commercialement à 1 $\mathrm{mg} / \mathrm{mL}$ en norkétamine. Les deux solutions mères sont stables pendant 3 mois, conservées à $-20^{\circ} \mathrm{C}$.

La solution mère d'ifosfamide à $2 \mathrm{~g} / \mathrm{L}$ (étalon interne) dans le méthanol est stable pendant 6 mois, conservée à $-20^{\circ} \mathrm{C}$. Une solution fille à $4 \mathrm{mg} / \mathrm{L}$ est préparée extemporanément par dilution au 1/500 de la solution mère dans de l'eau distillée.

Un pool de sérum «free » est utilisé pour la préparation de la gamme d'étalonnage et des contrôles. Il est obtenu à partir de sérum humain de patients non traités, agité à $+4{ }^{\circ} \mathrm{C}$ avec du charbon actif $(1 \mathrm{~g}$ pour $250 \mathrm{~mL})$ pendant 24 heures puis filtré (filtre Nalgène ${ }^{\circledR} 0,45 \mu \mathrm{m}$, VWR International, Fontenay-sous-Bois, France). Après filtration, le sérum « free » réparti en tubes de 5 $\mathrm{mL}$, est conservé au congélateur à $-20^{\circ} \mathrm{C}$.

\section{Système et conditions chromatographiques}

Les dosages de la kétamine et de son métabolite sont réalisés sur un chromatographe en phase gazeuse Hewlett Packard 5890 équipé d'un détecteur spécifique azote-phosphore (NPSD) et couplé à un système d'injection Hewlett Packard GC/SFC 7673. La colonne est une Hewlett Packard HP1 (longueur $30 \mathrm{~m}$, diamètre interne $530 \mu \mathrm{m}$, épaisseur $2,65 \mu \mathrm{m}$ ). L'hélium et utilisé comme gaz vecteur (débit $=7 \mathrm{~mL} / \mathrm{min}$ ). L'hydrogène et l'air utilisés comme gaz de combustion dans le détecteur, ont respectivement pour débit 1 et 70 $\mathrm{mL} / \mathrm{min}$. Les températures de l'injecteur et du détecteur sont de $250^{\circ} \mathrm{C}$ et de $275^{\circ} \mathrm{C}$. L'analyse est réalisée en isotherme à $205^{\circ} \mathrm{C}$. Le potentiel du détecteur est réglé de manière à obtenir une valeur de ligne base comprise entre 20 et 30 . Les données sont recueillies à l'aide d'un intégrateur (Hewlett Packard 3396 Series II integrator).

\section{Préparation de la gamme d'étalonnage et des contrôles}

Toutes les solutions et dilutions sont réalisées en tube borosilicatés de façon à limiter l'adsorption non spécifique des molécules à doser.

A partir des solutions mères de $\mathrm{K}$ et de $\mathrm{NK}$, des solutions filles sont préparées par dilution extemporanée au $1 / 20$ dans le chlorure de sodium isotonique, pour obtenir des solutions à $50 \mu \mathrm{g} / \mathrm{L}$ en $\mathrm{K}$ et en NK.

La gamme d'étalonnage en 6 points (500 à $4000 \mu \mathrm{g} / \mathrm{L}$ ) est préparée par surcharge du sérum « free » en $\mathrm{K}$ et NK. Des volumes croissants (5 à $40 \mu \mathrm{L}$ ) de chaque solution fille sont déposés dans des tubes borosilicatés.
Le volume est complété à $100 \mu \mathrm{L}$ avec du chlorure de sodium isotonique, puis $400 \mu \mathrm{L}$ de sérum « free » sont ajoutés à chacun des points de gamme. Le sérum «free » seul permet l'obtention du point zéro.

Trois contrôles de qualité sont réalisés par surcharge du même sérum à des concentrations basse $\left(\mathrm{CQ}_{1}\right.$ : $500 \mu \mathrm{g} / \mathrm{L})$ moyenne $\left(\mathrm{CQ}_{2}: 1800 \mu \mathrm{g} / \mathrm{L}\right)$ et haute $\left(\mathrm{CQ}_{3}\right.$ : $3600 \mu \mathrm{g} / \mathrm{L})$.

Les points de gamme et les contrôles sont répartis sous un volume de $100 \mu \mathrm{L}$ et congelés à $-20^{\circ} \mathrm{C}$. Dans ces conditions les échantillons sont stables pendant un mois.

\section{Extraction des échantillons}

A une prise d'essai de $100 \mu \mathrm{L}$ (gamme, contrôles et échantillons) est ajouté $200 \mu \mathrm{L}$ d'étalon interne (EI) à $4 \mathrm{mg} / \mathrm{L}$ ). Les échantillons sont alcalinisés par $50 \mu \mathrm{L}$ de soude $0,1 \mathrm{~N}$ puis extraits par $1,5 \mathrm{~mL}$ de chloroforme au multi vortex ( 3 fois 15 secondes). Après centrifugation 10 minutes à 4000 tours, $1,2 \mathrm{~mL}$ de phase organique est récupéré. L'extrait est évaporé à $37^{\circ} \mathrm{C}$ sous courant d'azote. Les extraits secs sont repris par $100 \mu \mathrm{L}$ d'acétate d'éthyle et $1 \mu \mathrm{L}$ est injecté dans le système chromatographique.

\section{Étude pharmacocinétique chez l'animal}

Pour la réalisation des cinétiques, des rats mâles Sprague-Dawley (Souche OFA, Charles River laboratoires, BP0109 69592 L'arbresle Cedex) de 250 à $350 \mathrm{~g}$ (8 à 12 semaines) ont été utilisés. Les animaux étaient livrés au minimum une semaine avant le jour de l'expérimentation. Ils sont gardés à l'animalerie dans des conditions de température et de luminosité adaptées, l'eau et la nourriture étant fournies ad libidum. Les animaux survivants à l'expérimentation sont euthanasiés au dioxyde de carbone.

La veille de l'expérimentation, les animaux sont anesthésiés avec du thiobarbital $(60 \mathrm{mg} / \mathrm{kg}$ par voie intrapéritonéale) pour la mise en place de cathéters au niveau de l'artère et de la veine fémorales.

Le jour de l'expérimentation les animaux reçoivent dans la veine fémorale, en perfusion de $30 \mathrm{~min}$, une dose de $70 \mathrm{mg} / \mathrm{kg}$ de kétamine (exprimée en kétamine base). Les échantillons de sang ( $200 \mu \mathrm{L})$ sont recueillis de façon fractionnée entre 0 et $330 \mathrm{~min}$ au niveau du cathéter de l'artère fémorale. Ils sont immédiatement centrifugés et le plasma est conservé à $-20^{\circ} \mathrm{C}$ jusqu'au moment du dosage.

\section{Expression des résultats et paramètres pharmacocinétiques}

Les résultats sont exprimés en moyenne $+/$ - écart- type $(\mathrm{m}+/$ - S.D. $)$

La modélisation de la cinétique et le calcul des para- 
mètres pharmacocinétiques obtenus après administration IV de la kétamine chez le rat, ont été déterminés en utilisant le logiciel WinNonlin ${ }^{\circledR}$ (Pharsight, USA).

\section{Résultats}

\section{Profils chromatographiques}

La figure 2 représente les tracés chromatographiques obtenus pour le point zéro (Figure 2a), un point de gamme à $1500 \mu \mathrm{g} / \mathrm{L}$ (Figure $2 \mathrm{~b}$ ) et un prélèvement obtenu chez le rat, correspondant à un temps 10 minutes après la fin de la perfusion (Figure 2c). Les pics obtenus sont fins, symétriques et bien séparés dans un temps d'analyse totale de 15 minutes. Les temps de rétention sont respectivement de $6,1,6,8$ et $9,4 \mathrm{~min}$, pour $\mathrm{NK}, \mathrm{K}$ et l'étalon interne.

\section{Validation analytique de la méthode}

Dans ce but, nous avons vérifié les critères habituels de validation que sont la linéarité, la limite de détection, la précision, l'exactitude et la spécificité.

\section{Linéarité}

La linéarité a été vérifiée en injectant trois gammes d'étalonnage le même jour dans les mêmes conditions chromatographiques. Le calcul des rapports surfaces des molécules d'intérêt (K et NK) sur EI, montrent que les relations sont linéaires pour la zone de concentrations comprises entre 500 et $4000 \mu \mathrm{g} / \mathrm{L}$. En utilisant la régression par la méthode des moindres carrés, les équations moyennes des droites d'étalonnage sont : NK $y=0,00032 x-0,012$ et $K$ y $=0,00020 x-0,020$ avec des coefficients de corrélation compris entre 0,95 et 0,99 . Le tableau I résume pour chaque point de gamme, les

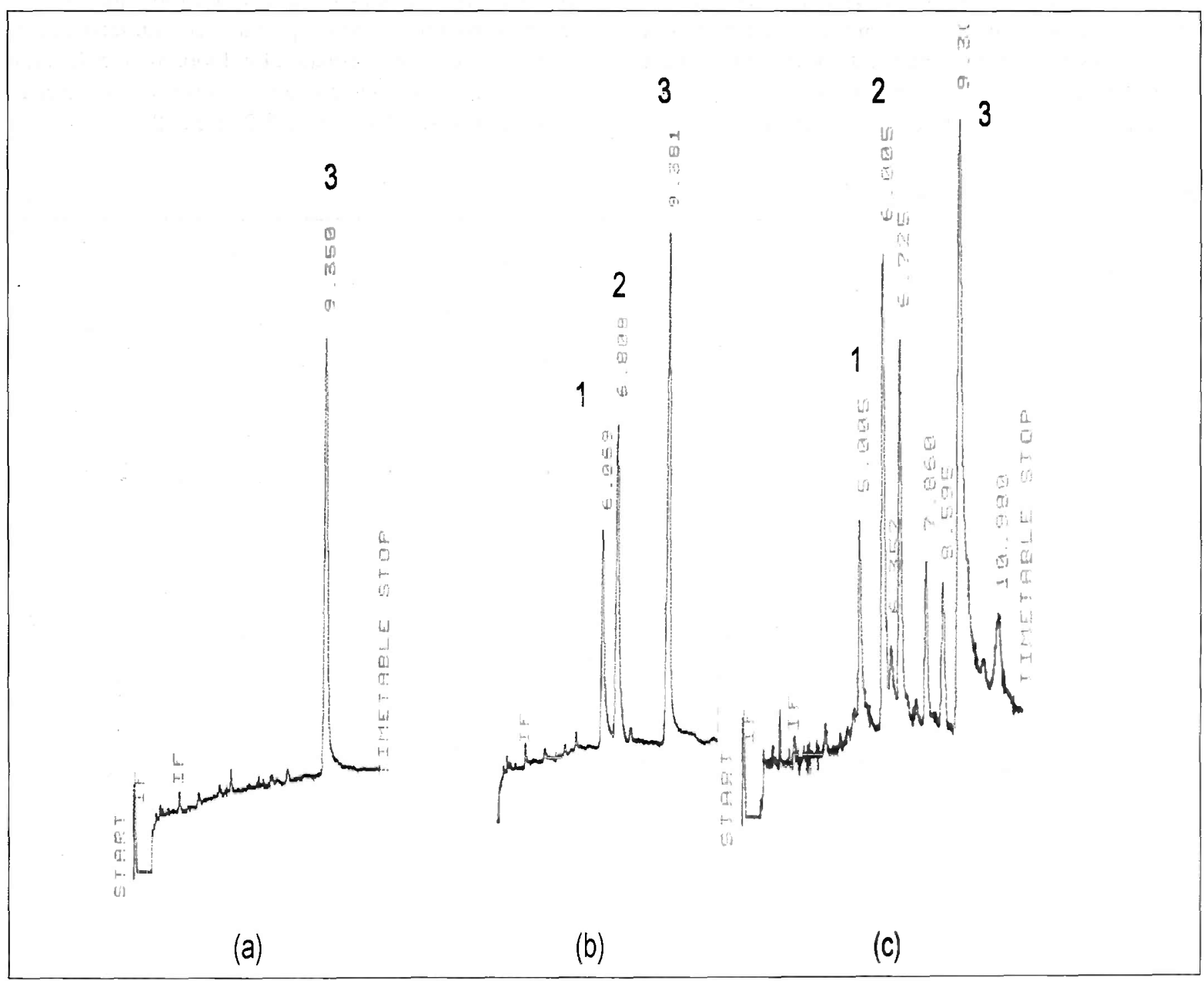

Figure 2 : Profil chromatographique du point zéro (a), d'un point de gamme à $1500 \mu \mathrm{g} / \mathrm{L}$ (b), et d'un prélèvement obtenu chez. le rat 10 minutes après la fin de la perfusion de kétamine (dose de $70 \mathrm{mg} / \mathrm{kg}$ ) (c). Pics :1= norkétamine ; $2=$ kétamine ; $3=$ ifosfamide (étalon interne). 
valeurs de la moyenne, des écart-types, de la précision (coefficient de variation) et de l'exactitude (biais).

\section{Limites de détection et de quantification}

La limite de détection (LD) a été déterminée en considérant un rapport signal/bruit de 3/1. Dans ces conditions elle est de $160 \mu \mathrm{g} / \mathrm{L}$ pour la kétamine et de $180 \mu \mathrm{g} / \mathrm{L}$ pour la norkétamine. Les limites de quantification (LQ) peuvent être considérées comme étant égales à un rapport signal/bruit de $10 / 1$, soit à des valeurs de LQ de 480 et $520 \mu \mathrm{g} / \mathrm{L}$ pour la K et la NK, respectivement. La limite de quantification pour les 2 molécules a donc été choisie à $500 \mu \mathrm{g} / \mathrm{L}$, correspondant au premier point de gamme.

\section{Exactitude et précision (inter- et intra-séries)}

Ces paramètres de validation ont été déterminés en mesurant les 3 contrôles de qualité. La précision de la méthode a été évaluée en inter ou en intra-séries $(\mathrm{n}=10)$ et exprimée en pourcentage de variation. Pour la kétamine et la norkétamine, les valeurs des coefficients de variation sont comprises entre 3,6 et 10,5\% (tableau II). Sur les mêmes déterminations, la valeur de l'exactitude a été calculée comme le pourcentage de biais observé par rapport à la valeur vraie. Les valeurs du biais sont toutes comprises dans les limites de plus ou moins $15 \%$ et sont donc compatibles avec une exactitude satisfaisante de notre méthode (tableau II).

\section{Rendement d'extraction}

Pour calculer le rendement d'extraction, nous avons comparé la hauteur des pics de solutions en K et NK préparées dans du méthanol à des plasmas surchargés aux mêmes concentrations. De façon à vérifier le rendement d'extraction sur une plage étendue de concentrations, nous avons retenu comme concentrations tests les valeurs des 3 contrôles de qualité, respectivement 500, 1800 et $3600 \mu \mathrm{g} / \mathrm{L}$. Sur 4 déterminations, nous avons observé un rendement d'extraction de $92+/-4 \%(500 \mu \mathrm{g} / \mathrm{L})$; $95+/-6 \%(1800 \mu \mathrm{g} / \mathrm{L})$ et $90+/-3 \%(3600 \mu \mathrm{g} / \mathrm{L})$.

\section{Spécificité}

Les chromatogrammes obtenus ne montrent aucune interférence biologique ou pharmacologique (Figure 2), en particulier avec les produits co-administrés lors de l'anesthésie des animaux. Le thiobarbital et la xylazine extraits dans les mêmes conditions sont détectés mais élués respectivement à $5,5 \mathrm{~min}$ et $8,3 \mathrm{~min}$.

Tableau I : Précision et exactitude de la gamme d'étalonnage.

\begin{tabular}{|c|c|c|c|c|}
\hline Composés & Concentrations théoriques $(\mu \mathrm{g} / \mathrm{L})$ & Concentrations calculées $(\mathrm{m}+/$-S.D., $\mathrm{n}=3)(\mu \mathrm{g} / \mathrm{L})$ & Précision $(\mathrm{C} . \mathrm{V} ., \%)$ & Exactitude $($ biais, \%) \\
\hline Norkétamine & 500 & $480+/-39$ & 8,1 & 10,3 \\
& 1000 & $1026+/-105$ & 11,3 & $-4,9$ \\
& 1500 & $1397+/-158$ & 9,0 & $-7,6$ \\
& 2000 & $1955+/-176$ & 7,0 & $-3,8$ \\
& 3000 & $3085+/-217$ & 6,7 & 1,8 \\
& 4000 & $3993+/-147$ & 10,9 & $-1,2$ \\
\hline Kétamine & 500 & $496+/-33$ & 11,9 & $-1,7$ \\
& 1000 & $1027+/-112$ & 9,4 & $-4,0$ \\
& 1500 & $1455+/-174$ & 5,4 & $-1,4$ \\
& 2000 & $1991+/-188$ & 2,7 & $-1,3$ \\
\end{tabular}

C.V. : coefficient de variation

Tableau II : Étude de la précision et de l'exactitude la méthode.

\begin{tabular}{|c|c|c|c|c|c|c|c|}
\hline \multirow[t]{2}{*}{ Composés } & \multirow{2}{*}{$\begin{array}{l}\text { Concentrations } \\
\text { théoriques }(\mu \mathrm{g} / \mathrm{L})\end{array}$} & \multicolumn{3}{|c|}{ Précision inter-séries $(\mathrm{n}=10)$} & \multicolumn{3}{|c|}{ Précision intra-séries $(n=10)$} \\
\hline & & $\begin{array}{l}\text { Concentrations mesurées } \\
(\mathrm{n}+/- \text { S.D. })(\mu \mathrm{g} / \mathrm{L})\end{array}$ & $\begin{array}{l}\text { Précision } \\
\text { (C.V., \%) }\end{array}$ & $\begin{array}{l}\text { Exactitude } \\
\text { (biais, \%) }\end{array}$ & $\begin{array}{c}\text { Concentrations mesurées } \\
(\mathrm{m}+/- \text { S.D. })(\mu \mathrm{g} / \mathrm{L})\end{array}$ & $\begin{array}{l}\text { Précision } \\
\text { (C.V., \%) }\end{array}$ & $\begin{array}{l}\text { Exactitude } \\
\text { (biais, \%) }\end{array}$ \\
\hline \multirow[t]{3}{*}{ Norkétamine } & 800 (CQI) & $732+1-56$ & 7.7 & 8.5 & $735+/-55$ & 7,5 & $-9,1$ \\
\hline & $1800(\mathrm{CQ} 2)$ & $1764+1-122$ & 6,9 & 2,0 & $1724+/-153$ & 8,9 & $-5,2$ \\
\hline & $3600(\mathrm{CQ} 3)$ & $3664+/-248$ & 6,8 & $-1,8$ & $3564+/-129$ & 3,6 & $-2,0$ \\
\hline \multirow[t]{3}{*}{ Kétamine } & $800(\mathrm{CQ} 1)$ & $767+1-71$ & 9,3 & 4,1 & $786+1-49$ & 6,2 & $-2,7$ \\
\hline & $1800(\mathrm{CQ} 2)$ & $1798+/-146$ & 8,1 & 0,1 & $1834+/-193$ & 10,5 & 0,9 \\
\hline & $3600(\mathrm{CQ} 3)$ & $3634+/-323$ & 8,9 & $-0,9$ & $3566+/-156$ & 4,4 & $-1,9$ \\
\hline
\end{tabular}

C.V. : coefficient de variation 


\section{Application à la pharmacocinétique de la kétamine chez le rat}

La figure 3 rapporte les cinétiques moyennes de $\mathrm{K}$ et NK obtenues chez 6 rats après perfusion de kétamine durant 30 minutes. Pour la kétamine, les concentrations plasmatiques sont très élevées dès le début de la perfusion, mais décroissent ensuite très rapidement selon une cinétique d'apparence biphasique. La formation de la norkétamine se produit dès le début de la perfusion. La modélisation pharmacocinétique montre qu'un modèle à 2 compartiments ouverts avec une cinétique d'élimination d'ordre 1 est parfaitement corrélé avec nos données expérimentales (Figure 4). Les principaux paramètres pharmacocinétiques de la kétamine calculés en utilisant ce modèle sont rapportés dans le tableau III. Nous retrouvons des demi-vies moyennes de 9,6 et 107

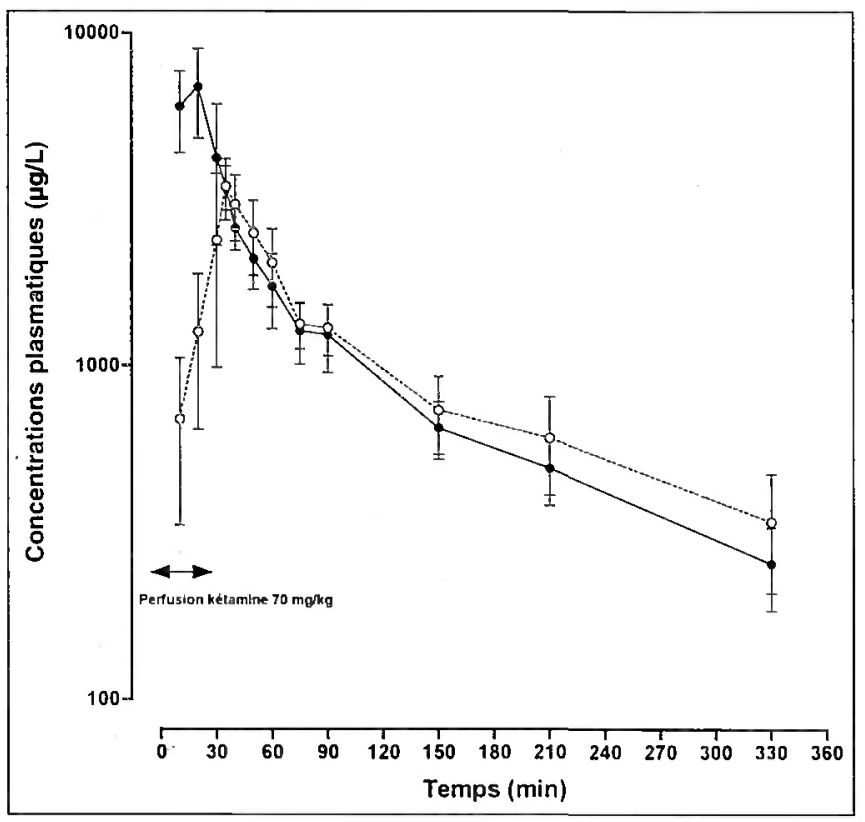

Figure 3 : Cinétiques en coordonnées semi-logarithmiques de la kétamine (cercle plein) et de la norkétamine (cercle clair) chez le rat male Sprague Dawley après perfusion d'une dose de kétamine $(70 \mathrm{mg} / \mathrm{kg})$ en 30 minutes. Les résultats sont représentés sous la forme $m+/-$ S.E.M. $(n=6)$. min, respectivement pour les phases de distribution et d'élimination. Le volume de distribution moyen est élevé de l'ordre de $19 \mathrm{~L} / \mathrm{kg}$. Le temps de séjour dans l'organisme (M.R.T.) est relativement court compatible avec une valeur très élevée de la clairance corporelle totale. Les constantes pharmacocinétiques sont caractérisées par une grande variabilité d'un animal à l'autre comme le montre la valeur des écart-types calculés pour chaque paramètre.

Pour la norkétamine, les concentrations vont croître pour passer par une valeur maximale cinq minutes après la fin de la perfusion, puis resteront supérieures à celles de la kétamine pendant toute la durée de l'étude. L'utilisation d'un modèle non compartimental montre une demi-vie d'élimination de 95 +/- 11 minutes comparable à celle rapportée dans la littérature $(11,15)$.

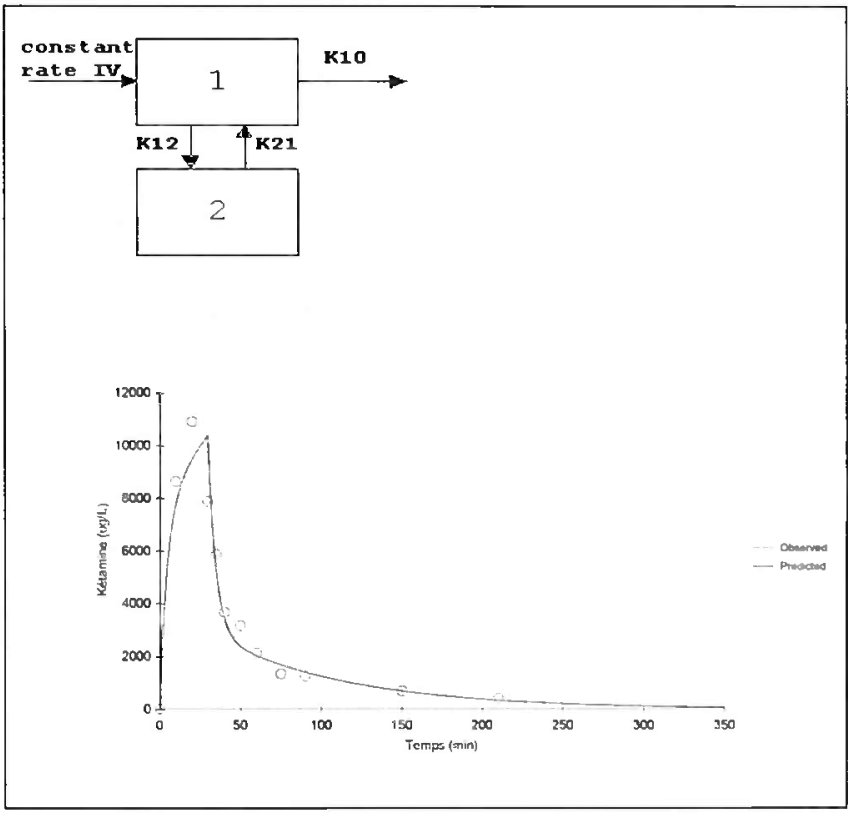

Figure 4: Modélisation de la cinétique de la kétamine après perfusion chez le rat.

Tableau III : Paramètres pharmacocinétiques moyens $(n=6)$ de la kétamine obtenus chez. le rat Sprague Dawley.

\begin{tabular}{|c|c|c|c|c|c|c|c|}
\hline & $\begin{array}{l}\text { Cmax } \\
\mu \mathrm{g} / \mathrm{L}\end{array}$ & $\begin{array}{c}\text { Demie vie } \\
\text { alpha } \\
\text { min }\end{array}$ & $\begin{array}{c}\text { Demie vie } \\
\text { béta } \\
\text { min }\end{array}$ & $\begin{array}{c}\text { A.U.C. } \\
\min ^{*} \mu \mathrm{g} / \mathrm{L}\end{array}$ & $\begin{array}{c}\text { CI } \\
\mathrm{L} / \mathrm{min} / \mathrm{kg}\end{array}$ & $\begin{array}{c}\text { Vd } \\
\mathrm{L} / \mathrm{kg}\end{array}$ & $\begin{array}{c}\text { M.R.T } \\
\min \end{array}$ \\
\hline moyenne & 4334 & 9.6 & 107 & 358216 & 0.2 & 19 & 95 \\
\hline S.E.M & 562 & 3.3 & 13 & 56026 & 0.03 & 4.8 & 11 \\
\hline
\end{tabular}

Cmax : concentration au pic plasmatique

A.U.C. : aire sous la courbe

$\mathrm{CI}$ : clairance totale

$\mathrm{Vd}$ : volume de distribution

M.R.T. : Mean residence time 


\section{Discussion}

Nous rapportons dans ce travail la mise au point d'une méthode de dosage de la kétamine $(\mathrm{K})$ et de son métabolite (NK) par chromatographie en phase gazeuse (CPG). La revue de la littérature montre que de nombreuses méthodes par CPG ont déjà été décrites permettant le dosage simultané de la kétamine et de la norkétamine (15) associé ou non à d'autres métabolites, comme la déhydronorkétamine (16). Ces méthodes utilisent toutes une étape de dérivation puis soit une détection non spécifique par ionisation (détecteur FID) ou plus généralement une détection azote-phosphore (détecteur NPD) afin d'accroître la sensibilité. Le laboratoire étant doté des deux modes de détection, nous avons comparé la sensibilité des détecteurs. Sans dérivation, le détecteur FID était 20 fois moins sensible que la détection NPD sur des solutions de kétamine ou de norkétamine à la concentration de $1000 \mu \mathrm{g} / \mathrm{L}$. Ces résultats sont similaires à ceux rapportés par Stiller et coll. (15) et Pedraz et coll. (16).

Les étapes de dérivation sont en général considérées comme consommatrices de temps, délicates à mettre en œuvre mais nécessaires pour améliorer la sensibilité de la détection. L'originalité de notre travail est de présenter une technique directe suffisamment sensible pour être appliquée à des prélèvements sériques sans étape préalable de dérivation. Cependant, à l'exception d'une seule publication (17), aucune technique décrite ne rapportait un dosage sans dérivation de ces composés. Nous avons donc testé une procédure de dérivation fondée sur l'utilisation des anhydrides d'acide comme le TFAA (anhydride trifluoroacétique) ou le HFBA (anhydride heptafluorobutyrique) (16). Dans notre expérience, les techniques de dérivation n ${ }^{\circ}$ ont pas montré d'amélioration significative de la sensibilité, contrairement à Siller et coll. (15) qui rapportent une augmentation de sensibilité pour la kétamine mais pas d'effet sur la norkétamine. L'absence d'amélioration de la sensibilité associée à une faible reproductibilité de la méthode de dérivation, nous a conduit à développer la méthode directe d'injection de l'extrait plasmatique.

L'étude des volumes d'échantillons montre des volumes élevés de plasma (supérieur à $0,5 \mathrm{ml}$ ) quelque soit la méthode chromatographique utilisée pour le dosage $(15,16,19,20)$. Un tel volume n'est pas compatible avec la réalisation de cinétiques chez l'animal, comme le rat ou le volume maximal pouvant être prélevé sur une cinétique complète est de l'ordre de $2 \mathrm{ml}$ de sang total. La méthode que nous avons développée permet le dosage de la $\mathrm{K}$ et de la $\mathrm{NK}$ à partir d'une prise d'essai de $100 \mu \mathrm{l}$ voire de $50 \mu \mathrm{l}$ pour les points les plus concentrés. Dans le but de travailler sur la prise d'essai la plus faible, nous avons comparé les méthodes d'extraction. La revue de la littérature rapporte que l'extraction liquide-liquide est largement la plus utilisée $(15,16,21)$ avec des solvants variés (éther, heptane, cyclohexane) $(4,15,21)$ ou des mélanges de solvants comme le mélange dichlorométhane-acétate d'éthyle (19). L'extraction solide-liquide basée sur l'utilisation de cartouche SPE C18 a également rapportée par d'autres auteurs $(11,20)$. Nous avons développé au laboratoire une extraction liquide-liquide basée sur l'utilisation d'un seul solvant, le chloroforme en milieu alcalin. Dans ces conditions, nous avons obtenu un rendement d'extraction très satisfaisant (>90\%) tant pour les molécules d'intérêt que pour l'étalon interne. Ces rendements sont comparables à ceux rapportés dans la littérature (4). Dans notre expérience, l'extraction par l'acétate d'éthyle conduit à des rendements similaires, mais aboutit à des profils chromatographiques présentant plus de pics non spécifiques tandis que l'extraction solide-liquide a conduit à des rendements inférieurs voisins de $80 \%$. Ce pourcentage est comparable à l'étude de Licata et coll. (13).

La plupart des techniques décrites $(15,20,21)$ utilisent comme étalon interne la bromokétamine, qui n'est pas disponible commercialement et ne peut plus être obtenue auprès des laboratoires synthétisant la kétamine. Nous avons testé les étalons rapportés pour des méthodes en phase liquide comme la désipramine (4) ou la nortidiline (19). Dans nos conditions chromatographiques, ces composés ne sont soit pas élués (nortidiline) soit élués trop tardivement pour la désipramine (tr $=15,3 \mathrm{~min}$ ) par rapport aux substances d'intérêt. L'étalon interne que nous avons retenu est l'ifosfamide, un antimitotique qui peut être extrait avec un rendement satisfaisant et chromatographié sans dérivation dans nos conditions. L'élution se fait avec un temps de rétention différent de ceux de la $\mathrm{NK}$ et de la $\mathrm{K}$ sans en être trop éloigné. Bien que l'ifosfamide soit un médicament antimitotique, son utilisation comme EI ne pose pas de problème pour les études chez l'animal, et pour les études chez l'homme, sa co-administration avec la kétamine reste fort peu probable.

Sur le plan de la sensibilité, nous avons déterminé une LQ voisine de $500 \mu \mathrm{g} / \mathrm{L}$ pour la $\mathrm{K}$ et son métabolite. Cette sensibilité est suffisante pour permettre en routine la détection des 2 molécules dans les études pharmacocinétiques chez l'homme comme chez l'animal. Dans un cadre de médecine légale, compte tenu de sa sensibilité, notre méthode ne pourrait que valider une prise récente de $\mathrm{K}$, mais ne saurait être suffisante pour les cas de soumission chimique, ou la spectrométrie de masse en tandem devrait lui être préférée.

L'étude de la spécificité de notre méthode s'est limitée 
à tester l'interférence du pentobarbital et de la xylazine utilisés pour le pré-traitement des rats dans notre protocole opératoire. Ces deux substances sont extraites, peuvent être chromatographiées dans nos conditions, mais présentent des temps de rétention différents des substances d'intérêt. Pour appliquer notre méthode chez l'homme, une étude plus exhaustive de produits potentiellement interférents, en particuliers les produits à action centrale (benzodiazépines, barbituriques,..) devrait être testés. Ces composés sont en effet susceptibles d'être consommés en mélange lors des « rave party »

Différents travaux ont rapportés l'existence d'une mauvaise conservation des solutions de $\mathrm{K}$ et de NK même à très basse température (22). Sur des périodes de conservation de durée moyenne $\left(<15\right.$ jours) à $+4^{\circ} \mathrm{C}$, nous n'avons pas observé de variations de concentrations aussi bien pour les solutions filles que pour des points de gamme à condition de conserver les solutions en tubes borosilicaté. Avec du verre non traité, nous avons observé une absorption non spécifique pouvant atteindre 20 à $30 \%$ et apparaissant dans les 24 heures suivant la préparation des solutions.

Nous avons appliqué cette méthode à une étude pharmacocinétique chez le rat après injection intraveineuse de kétamine. Les paramètres cinétiques ainsi que l'élimination biphasique sont comparables à ceux rapportés chez l'homme $(5,17)$ ou dans d'autres espèces animales $(4,22)$.

\section{Conclusion}

Nous rapportons dans ce travail une méthode de dosage de la kétamine et de son métabolite principal, la norkétamine, par chromatographie en phase gazeuse. Le principal intérêt de notre méthode provient d'une sensibilité satisfaisante obtenue en l'absence de dérivation. Notre méthode est donc facile à mettre œuvre, robuste et fiable. Applicable aux études pharmacologiques ou toxicologiques chez l'homme, notre méthode trouve son principal intérêt dans les protocoles pharmacocinétiques chez le petit animal en raison du faible volume de prélèvement nécessaire à sa mise en œuvre.

\section{Remerciements}

Les auteurs adressent tous leurs remerciements à Mesdames M. Corvisier et I. Souletie pour leur précieuse aide technique dans la réalisation des dosages et au Dr. M. Debray pour l'analyse des paramètres pharmacocinétiques.

\section{Références}

1. Cheng J.Y.K., Mok V.K.K. Rapid determination of ketamine in urine by liquid chromatography-tandem mass spectrometry for a high throughput laboratory. Forensic. Sci. Int. $2004 ; 142$ : 9-15.

2. Riou B., Langeron O. La kétamine. In : Gosgnach M., Chauvin, M., Riou B., eds. Pharmacologie en anesthésieréanimation. Paris : Arnette, 1999 ; 287-301.

3. White P.F., Way W.L., Trevor, A.J. Ketamine-its pharmacology and therapeutic use. Anaesthesiology $1982 ; 56$ : 119-136.

4. Niedorf F., Bohr H.H., Kietzmann M. Simultaneous determination of ketamine and xylazine in canine plasma by liquid chromatography with ultraviolet absorbance detection. J. Chromatogr. B. 2003 ; 791 : 421-426.

5. Wieber J., Gugler R., Hengstmann J.H., Dengler H.J. Pharmacokinetics of ketamine in man. Anaesthetist $1975 ; 24: 260-263$.

6. Hijazi Y., Boulieu R. Protein binding of ketamine and its active metabolites to human serum. Eur. J. Clin. Pharmacol. $2002 ; 58$ : 37-40.

7. Adams J.D., Baillie T.A., Trevor A.J., Castagnoli N. Studies on the biotransformation of ketamine.1. Identification of metabolites produced in vitro from rat liver microsomal preparations. Biomed. Mass Spectrom $1981 ; 8: 527-538$.

8. Martin D., Lodge D. Ketamine acts as a non competitive $\mathrm{N}$-methyl-D-aspartate antagonist on frog spinal cord in vitro. Neuropharmacology $1985 ; 10$ : 999-1003.

9. Graf M.B., Vicenzi M.N., Martine E., Bosnjak Z.J., Stowe D.F. Ketamine has stereospecific effects in the isolated perfused guinea pig heart. Anaesthesiology 1995 ; 82 : 1426-1437.

10. Bjarke E., Mikkelsen S., Thorkildsen C., Borgbjerg F.M. Norketamine, the main metabolite of ketamine, is a non competitive NMDA receptor antagonist in the rat cortex and spinal cord. Eur. J. Pharmacol. $1997 ; 333$ : 99-104.

11. Hijazi Y., Bolon M., Boulieu R. Stability of ketamine and its metabolites norketamine and dehydronorketamine in human biological samples. Clin. Chem. $2001 ; 47$ (9) : 1713-1715.

12. Dalgarno P.J., Shewan D. Illicit use of ketamine in Scotland. J Psychoactivre Drugs 1996 ; 28 : 2 : 191-199.

13.Licata M., Pierini G. A fatal ketamine poisoning. J. Forensic. Sci $1994: 39: 1314-1320$.

14. Gill J.R., Stajic M. Ketamine in non-hospital and hospital deaths in New York City. J. Forensic. Sci $2000 ; 45$ : $3: 655-658$.

15.Stiller R.L., Dayton P.G., Perel J.M. Gas chromatographic analysis of ketamine and norketamine in plasma and urine: nitrogen-sensitive detection. J. Chromatogr. 1982 ; 232 : 305-314.

16.Pedraz J.L., Marino E.L., Dominguez-Gil A. Quantitative determination of ketamine and its metabolites in man by gas-liquid chromatography. Il Farmaco; $38-5: 209-218$. 
17. Malinovsky J.M., Servin F., Cozian A., Lepage J.Y., Pinaud M. Ketamine and norketamine plasma concentrations after i.v., nasal and rectal administration in children. Br. J. Anaesth. $1996 ; 77$ : 203-207.

18. Kochhar M.M. The identification of ketamine and its metabolites in biologic fluids by gas-chromatographymass spectrometry. Clin. Toxicol. $1991 ; 67$ : 310-316.

19. Bolze S., Boulieu R. HPLC determination of ketamine, norketamine, and dehydronorketamine in plasma with a high-purity reversed-phase sorbent. Clin. Chem. 1998 ; $44: 3: 560-564$.

20.Rodriguez Rosas M.E., Patel S., Wainer I.W. Determination of the enantiomers of ketamine and norketamine in human plasma by enantioselective liquid chromatography- mass spectrometry. J. Chromatogr. B. $2003 ; 794: 99-108$.

21. Yanagihara Y., Ohtani M., Kariva S., Uchino K., Aoyama T., Yamamura Y., Iga I. Stereoselective high-performance liquid chromatographic determination of ketamine an dits active metabolite, norketamine in human plasma. J. Chromatogr. B. $2000 ; 746: 227-231$.

22. Waterman A.E., Robertson S.A., Lane J.G. Pharmacokinetics of intravenously administered ketamine in the horse. Res Vet Sci. $1987 ; 42$ (2) : 162-166. 